\section{Influence of normal mammary epithelium on breast cancer progression: the protective role of early pregnancy}

\author{
Filipe Correia Martins $s^{1,2,3,4, *}$, Maria Filomena Botelho 2,3 , \\ António Manuel Cabrita ${ }^{2,4}$, and Carlos Freire de Oliveira ${ }^{1,2}$ \\ ${ }^{1}$ Department of Gynecology, University Hospital of Coimbra; ${ }^{2}$ CIMAGO (Oncobiology, Genetics and \\ Environment Research Center), Coimbra Medical School; ${ }^{3}$ Biophysics and Biomathematics Institute, \\ IBILI, Coimbra Medical School; ${ }^{4}$ Experimental Pathology Institute, Coimbra Medical School, \\ Coimbra, Portugal. *Current affiliation: Dana-Farber Cancer Institute, Harvard Medical School, \\ Boston, USA; Gulbenkian Program for Advanced Medical Education
}

\section{ABSTRACT}

\begin{abstract}
Aims and background. The microenvironment has a well recognized role in breast cancer progression. Despite different theories, the mechanism of early pregnancy protection in mammary carcinogenesis is unknown. Since pregnancy is responsible for mammary gland differentiation, we tested the hypothesis that differentiated mammary epithelial cells may inhibit breast cancer progression. In other words, the protective role of early pregnancy could be due to the inhibitory influences of the more differentiated mammary tissue.
\end{abstract}

Methods. In order to test our hypothesis, we used 30 female Balb/c nude mice and MCF-7 cells of breast adenocarcinoma. The female mice were divided into two test groups, group I (GI) and group II (GII), and a control group. In GII, the animals were submitted to epithelial removal in the left fourth inguinal mammary gland at 3 weeks of age. Both groups were given continuous hormonal treatment to simulate the pregnancy development of the mammary gland. Two million MCF-7 cells were then injected into the fourth inguinal mammary gland (GI) or in the respective cleared mammary fat pad (GII). Five weeks later the mice were sacrificed and their tumors removed. Tumor development rates and tumor volumes were determined and proliferation and apoptosis were evaluated by immunohistochemistry.

Results. Tumors of GII mice had a larger mean volume than those of GI mice ( $P=$ 0.001 , Mann-Whitney $U$-test) and an apparent increase in proliferation, demonstrated by a higher staining intensity for proliferating cell nuclear antigen (PCNA). As tumors presented caspase 8 staining, there may be apoptotic activation involved in cell death, mainly through an extrinsic pathway.

Conclusions. These results suggest that a differentiated intact mammary gland may have an inhibitory influence on mammary tumor growth in mice. Free full text available at www.tumorionline.it

\section{Introduction}

Tumor formation can be prevented by suppressing carcinogenesis or blocking tumor promotion. In experimental models, administration of a combination of antioxidant micronutrients has been correlated with a protective effect in breast cancer, especially due to a decreased incidence of tumors with a better prognosis ${ }^{1-3}$. Prevention of mammary carcinogenesis can also be achieved by pregnancy or by administration of hormones such as progesterone, estrogen and human chorionic gonadotropin in animal models ${ }^{4,5}$.

Although there are several epidemiological parameters which may influence breast
Key words: breast cancer, pregnancy, mammary epithelium.

Acknowledgments: We would like to thank Prof João Patrício and Prof José Luís Santos for their background support in microsurgery and statistical analysis of the results, respectively. We would also like to thank Dr Hanna Guimarães for careful reading and language revision of the manuscript, and Mrs Margarida Menezes, Mrs Elisa França, Dr Pedro Peça, Dr Margarida Abrantes and $\mathrm{Dr}$ Mafalda Laranjo for other technical support.

Correspondence to: Filipe Correia Martins, MD, Dana Farber Cancer Institute, D740, 44 Binney St, Boston, MA 02115, USA.

Tel +1-617-301-3518;

e-mail

filipe_martins@dfci.harvard.edu;

filipecorreiamartins@gmail.com

Received September 2, 2009;

accepted July 8, 2010. 
cancer incidence, early full-term pregnancy is considered by many authors as one of the most important factors in the decrease of the lifetime breast cancer risk ${ }^{4,6}$. Women bearing a child before the age of 20 have an approx. $50 \%$ lower risk of developing breast cancer than other women. However, a recent meta-analysis demonstrated that only hormone-responsive breast tumors are susceptible to the beneficial effects of parity ${ }^{7}$. It has also been shown that pregnancy induces a unique genomic signature in the epithelial compartment of the human breast $^{8}$. In this study, stroma was removed by microdissection, although different cellular populations were not considered in the epithelial compartment. These permanent changes in gene expression were also demonstrated in hormonally treated rats ${ }^{9}$.

Four main theories have been put forward to explain the protective role of pregnancy. According to the first theory, hormonal changes, namely in estradiol, prolactin and growth hormone levels, are responsible for the parity protection ${ }^{10}$. In animal models, both prolactin and growth hormone are reduced in parous compared to nulliparous animals after adjustment for age. These hormonal changes might result in a lower expression of estrogen receptors (ER) and epidermal growth factor receptors in epithelial cells, decreasing their carcinogenic susceptibility. The second theory focuses on the role of cell differentiation, which is maintained after involution, and hypothesizes that luminal cells may have a decreased ability to proliferate, hence becoming less susceptible to carcinogenic stimuli ${ }^{11}$. The third hypothesis is based on cancer stem cell theory ${ }^{12,13}$. It states that pregnancy decreases the number of mammary stem cells, which in turn are potentially susceptible to malignant transformation ${ }^{14}$, although recent studies by the same group have shown the opposite in mature adult mice ${ }^{15}$. Finally, since the protection due to pregnancy is specific for ER-positive tumors, the last theory considers that it may be mediated by changes in estrogen responsiveness, in a direct or paracrine way, through ER-dependent or ER-independent mechanisms ${ }^{7}$. All 4 theories focus on the decreased susceptibility of mammary epithelial cells to carcinogenic transformation after pregnancy, but none of them considers the differentiated epithelium itself as being able to inhibit tumor progression, even if the cells maintain their ability to initiate the carcinogenic process.

Some authors have stressed the importance of microenvironment and extracellular matrix remodeling during pregnancy and involution in the breast cancer risk $^{16}$. A key question that is still unanswered is whether the refractoriness is intrinsic to the mammary epithelial cells and/or mediated by persistent alterations in the host environment ${ }^{5}$.

A previous study revealed that, when epithelial cells of mammary glands from virgin female rats were exposed to a potent carcinogen like MNU (methylnitrosourea) and then transplanted into pregnant female rats, the tu- mor incidence in the pregnant rats was lower than in the host virgin female rats ${ }^{17}$. This study demonstrated the relevance of the surrounding environment in carcinogenesis, although it failed to establish if it only inhibits cell initiation or is able to inhibit tumor growth. Authors from the same group recently published another study using a similar animal model approach to demonstrate that mammary cancer development can be blocked by inhibiting or blocking promotion and progression of carcinogen-initiated cells (not directly affecting initiation) and that this may be related to the protection conferred by an early pregnancy ${ }^{18}$. In our study we therefore tested the hypothesis that differentiated mammary epithelium by an early pregnancy may inhibit breast cancer growth and progression.

To approach our hypothesis, we used previously developed models of pregnancy in mice and determined the influence of the differentiated intact mammary gland (compared to the cleared mammary fat pad) in tumor growth after the injection of tumor cells.

\section{Material and methods}

Thirty female Balb-c nude mice were used in this research, 20 in 2 test groups and 10 in a control group. All experimental procedures performed in the mice were approved by the National Animal Care Institute and were according to institution guidelines. Animals were maintained in laminar flow rooms under constant temperature and humidity on a 12-hour light, 12-hour dark cycle. Sterile laboratory chow and water were available ad lib. Surgical procedures were performed under sterile conditions.

At 3 weeks of age, 20 mice were randomly allocated to 2 groups: group I (GI; $\mathrm{n}=10$ ) and group II (GII; $\mathrm{n}=10$ ). GII mice were submitted to surgical removal of the epithelium of the fourth inguinal mammary gland by a previously described procedure with slight modifications ${ }^{19}$.

When the mice were 8 weeks old, estrogen $(400 \mu \mathrm{g} / \mathrm{kg})$ and progesterone $(40 \mathrm{mg} / \mathrm{kg})$ lipid solutions were administered daily for 21 days by dorsal subcutaneous injections in both groups to simulate the pregnancy development of the mammary gland ${ }^{20}$. Two million MCF7 cells (purchased from ATCC) were injected into the fourth inguinal mammary gland (GI) or the respective cleared mammary fat pad (GII) when the mice were 11 weeks old. Mice received estradiol benzoate supplementation $(1 \mathrm{mg} / \mathrm{kg}$ s.c.) every week after cell injection. These experiments were controlled for hormonal administration by the use of a control group of 10 mice with non-hormone-stimulated intact mammary glands.

Mouse weight and tumor development were measured weekly. Five weeks after cell injection, the mice were sacrificed by cervical dislocation. Macroscopic tumors were excised and tumor volumes calculated using 
the following equation: $\mathrm{V}=\left(\mathrm{a}^{2 *} \mathrm{~b}\right) / 2$, where $\mathrm{V}$ represents the tumor volume, a the larger axis and $b$ the shorter axis of the tumor.

Tissue fragments were fixed in $10 \%$ phosphatebuffered formalin and embedded in paraffin. Five- $\mu \mathrm{m}$ sections were obtained from the paraffin blocks and stained with hematoxylin and eosin for histological examination. Immunohistochemistry was performed for PCNA (Lab Vision), caspase 3 (Lab Vision - proenzyme not cleaved) and caspase 8 (Affinity BioReagents) according to a simple 2-step visualization system of very high sensitivity (Dako Envision Systems - kits K4010 and K4012, for rabbit and mouse primary antibodies, respectively). We used heat-induced antigen retrieval with pH 6 citrate buffer. Tumors with strong staining of PCNA and caspase 8 and tonsillar tissues, which stain strongly for caspase 3 , were used as controls for the immunochemical analyses. A blind study was performed by 2 pathologists to obtain the immunohistochemistry results. Immunostaining areas were calculated using informatics tools and PCNA immunostaining intensities were evaluated by comparison with a control tumor using a 0 - 3 scale $(0-$ no staining; 1 - lower than control; 2 - equal to control; 3 - higher than control) ${ }^{21}$.

Tumor volumes and areas of immunostaining were statistically evaluated with the Mann-Whitney $U$-test. The number of developed tumors was analyzed using Fisher's exact test. $P$ values $<0.05$ were considered significant.

\section{Results}

Nine mice, 3 in each group, were excluded from the study because of early death caused by technical errors. The remaining mice had no significant weight differences. In each tumor-bearing mouse, only 1 tumor appeared at the graft site. In GI, 4 mice (57\%) developed tumors, while all GII mice ( $P=0.096$, Fisher's test $)$ and 3 control group mice (43\%; $P=0.5$, Fisher's test) developed tumors. The mean tumor volume (MTV, calculated taking into account mice with no tumors) was $5.4 \mathrm{~mm}^{3}$ and $56.9 \mathrm{~mm}^{3}$ in GI and GII, respectively ( $P=0.001$, MannWhitney $U$-test). Similar differences were found when the mean tumor volume was calculated in tumor-bearing mice only. Control group tumor volumes were not statistically different from GI tumor volumes (Table 1).

All tumors in both test groups had a high proliferation index (more than $50 \%$ of tumor cells stained with PCNA) as evaluated by immunohistochemistry. The staining percentages were not significantly different between the 2 test groups $(P=0.382$, Mann-Whitney $U$-test). However, the immunostaining intensity was higher in GII than GI (Table 2). There were no significant differences in immunostaining intensity between GI and the control group.

Caspase 8 and caspase 3 immunostaining was identical between groups. About half of the cells in each tu-
Table 1 - Evaluation of final weight, number of tumor-presenting mice and mean tumor volumes

\begin{tabular}{llll}
\hline & Group I & Group II & Control group \\
\hline Weight of mice $(\mathrm{g})$ & 22.7 & 21.5 & 21.7 \\
Tumor-presenting mice $(\%)$ & $4 / 7(57 \%)$ & $\begin{array}{l}7 / 7(100 \%) \\
(P=0.096)^{*}\end{array}$ & $\begin{array}{l}3 / 7(43 \%) \\
(P=0.5)^{*}\end{array}$ \\
& & $\begin{array}{l}56.9 \\
(P=0.001)^{\circ}\end{array}$ & $2.7(P=0.155)^{\circ}$ \\
MTV $\left(\mathrm{mm}^{3}\right)$ in all mice & 5.4 & $\begin{array}{l}56.9 \\
(P=0.003)^{\circ}\end{array}$ & $7(P=0.086)^{\circ}$ \\
MTV $\left(\mathrm{mm}^{3}\right)$ & 9.5 & &
\end{tabular}

*Fisher test. ${ }^{\circ}$ Mann-Whitney $U$-test. MTV, mean tumor volume.

Table 2 - PCNA immunostaining intensities

\begin{tabular}{lllll}
\hline & 0 & 1 & 2 & 3 \\
\hline Group I & - & 1 & 2 & 1 \\
Group II & - & - & 2 & 5 \\
Control group & - & 1 & 1 & 1 \\
\hline
\end{tabular}

PCNA, proliferating cell nuclear antigen. 0 , no staining; 1 , lower than control; 2 , equal to control; 3 , higher than control.

mor stained for caspase 8 and only a few tumors presented vestigial staining for caspase 3 , despite its positivity in the control group (data not shown).

\section{Discussion}

Tumor heterogeneity arises from different mutations and tumors are unique and complex entities not susceptible to inhibitory or antigrowth stimuli. Nevertheless, based on previous studies ${ }^{17,18}$, we hypothesized that a differentiated normal mammary epithelium may itself inhibit breast cancer progression. Some recent in vitro studies also state that normal mammary epithelial cells exert an inhibitory effect via paracrine pathways ${ }^{22}$.

We have improved on a previously used experimental model by surgically removing the mammary epithelium and leaving the remaining cleared mammary fat pad intact for receiving cells or tissue transplants. The estradiol and progesterone administered in this experiment are able to simulate the development of a pregnant gland. There was a lag time between the surgical procedure, hormone administration, and cell injection, to make sure that surgery did not interfere in tumor growth.

All mice of GII but only some of GI developed tumors. The tumors of GII mice were also larger than those of GI mice. Within a single tumor cell line, there is a certain phenotypic variability due to epigenetic influences. Some of these variants may be more resistant to the proposed inhibitory effect and this may explain the appearance of a few small tumors in GI. By injecting the same number of tumor cells from the same cell line in 
an intact mammary gland and a cleared mammary fat pad and obtaining different tumor outcomes, we demonstrated that the differentiated mammary gland may inhibit tumor growth. Tumors of control group mice with non-hormone-stimulated intact mammary glands presented no statistical difference when they were compared with tumors of GI mice. This suggests that epithelial cells in general (or at least a subpopulation of them) may have these inhibitory effects, and that their number and position within the mammary gland after pregnancy may be an important factor in its protection.

There are some limitations to this study which should be mentioned and discussed. Despite the lag time between surgery and other procedures, a sham operation should have been performed in GII mice to avoid bias related to wound healing, which is known to promote tumor growth. Moreover, we used a single ER-presenting cell line in this study, because pregnancy protection seems to be specific for ER-positive tumors. However, other breast cancer cell lines expressing different receptors should be tried in the future. Finally, we sought to address the question of whether the normal mammary epithelium itself may inhibit tumor promotion. However, by removing the epithelium in very young mice, the stroma and extracellular matrix may have developed differently. Thus, we did not discard the role of the microenvironment in tumor growth.

A hypothetic explanation for the inhibition may be related to the normal differentiation process of the mammary gland. Progenitor cells proliferate and differentiate in a limited way and, when there is a functionally sufficient contingent of terminally differentiated cells, progenitor cells should be susceptible to inhibitory paracrine signals (cytokines) derived from the same differentiated cells. Taking into account well-known genetic and phenotypic similarities between tumor and progenitor cells ${ }^{13}$, we may consider that inhibitory pathways derived from normal differentiated cells may be preserved and still have an effect on tumor progression. Further experiments in normal mammary gland differentiation will contribute towards a better understanding of tumor-epithelium interactions.

In this study we do not refute previous theories concerning the role of pregnancy in protecting against breast cancer, although we have demonstrated that the influence of normal mammary epithelium in breast cancer progression should also be taken into consideration for a complete approach to the study of this role. By administering the same amount of hormones in both groups, we showed that hormonal changes on their own cannot account for the parity protection ${ }^{10}$. By injecting tumor cells, we demonstrated that although there are changes in mammary gland properties during pregnancy, its protective role also cannot be explained solely by lower susceptibility to carcinogenic stimuli of parous mammary cells ${ }^{11,14}$.
The previously referred concepts may be integrated in the initiation/promotion/progression model of cancer, considering either cancer stem cells or clonal evolution models ${ }^{23,24}$. A mutation in a stem cell or progenitor cell would initiate the process. However, this mutated cell would be controlled by the equilibrium between stimulatory and inhibitory factors. If this equilibrium were lost (for example, in chronic inflammation or other pathological conditions that destroy the normal differentiated environment, thus removing tumor inhibition), the promotion phase would begin. Later, the accumulation of further mutations and phenotypic changes in tumor cells would permit tissue invasion and systemic dissemination characteristic of the progression phase.

Breast cancer promotion and progression are blocked by previous pregnancies. According to our results, this blocking may be also due to the inhibitory influence of the differentiated mammary epithelium. Further insight into this influence may elucidate the protective role of pregnancy in breast cancer, and this will aid in the development of new prevention and treatment strategies.

\section{References}

1. Martins FC, Teixeira F, Reis I, Geraldes N, Cabrita AM, Dias MF: Chemoprevention and mammary neoplastic aggresiveness. Breast J, 14: 608-609, 2008.

2. Martins FC, Teixeira F, Reis I, Geraldes N, Cabrita AM, Dias MF: Increased transglutaminase 2 and GLUT- 1 expression in breast tumors not susceptible to chemoprevention with antioxidants. Tumori, 95: 227-232, 2009.

3. Dias MF, Sousa E, Cabrita S, Patricio J, Oliveira CF: Chemoprevention of DMBA-induced mammary tumors in rats by a combined regimen of alpha-tocopherol, selenium, and ascorbic acid. Breast J, 6: 14-19, 2000.

4. Martins FC, Botelho MF, Laranjo M, Cabrita AM, Torgal I, de Oliveira CF: Pregnancy and its role in breast cancer. Oncol Rev, 2: 141-145, 2008.

5. Sivaraman L, Medina D: Hormone-induced protection against breast cancer. J Mammary Gland Biol Neoplasia, 7: 77-92, 2002.

6. Henderson BE, Powell D, Rosario I, Keys C, Hanisch R, Young M, Casagrande J, Gerkins V, Pike MC: An epidemiologic study of breast cancer. J Natl Cancer Inst, 53: 609-614, 1974.

7. Britt K, Ashworth A, Smalley M: Pregnancy and the risk of breast cancer. Endocr Relat Cancer, 14: 907-933, 2007.

8. Balogh GA, Heulings R, Mailo DA, Russo PA, Sheriff F, Russo IH, Moral R, Russo J: Genomic signature induced by pregnancy in the human breast. Int J Oncol, 28: 399-410, 2006.

9. Blakely CM, Stoddard AJ, Belka GK, Dugan KD, Notarfrancesco KL, Moody SE, D'Cruz CM, Chodosh LA: Hormone-induced protection against mammary tumorigenesis is conserved in multiple rat strains and identifies a core gene expression signature induced by pregnancy. Cancer Res, 66: 6421-6431, 2006.

10. Henderson BE, Feigelson HS: Hormonal carcinogenesis. Carcinogenesis, 21: 427-433, 2000.

11. Russo J, Tay LK, Russo IH: Differentiation of the mammary 
gland and susceptibility to carcinogenesis. Breast Cancer Res Treat, 2: 5-73, 1982.

12. Trosko JE, Chang CC: Stem cell theory of carcinogenesis. Toxicol Lett, 49: 283-295, 1989.

13. Martins FC, Botelho MF, Cabrita AM, Torgal I, CF de Oliveira: Cancer and deregulation of stem cells pathways. Oncol Rev, 2: 199-202, 2008.

14. Smalley M, Ashworth A: Stem cells and breast cancer: a field in transit. Nat Rev Cancer, 3: 832-844, 2003.

15. Britt KL, Kendrick H, Regan JL, Molyneux G, Magnay FA, Ashworth A, Smalley MJ: Pregnancy in the mature adult mouse does not alter the proportion of mammary epithelial stem/progenitor cells. Breast Cancer Res, 11: R20, 2009.

16. McDaniel SM, Rumer KK, Biroc SL, Metz RP, Singh M, Porter W, Schedin P: Remodeling of the mammary microenvironment after lactation promotes breast tumor cell metastasis. Am J Pathol, 168: 608-620, 2006.

17. Abrams TJ, Guzman RC, Swanson SM, Thordarson G, Talamantes F, Nandi S: Changes in the parous rat mammary gland environment are involved in parity associated protection against mammary carcinogenesis. Anticancer Res, 18: 4115-4121, 1998.

18. Lakshmanaswamy R, Guzman RC, Nandi S: Hormonal prevention of breast cancer: significance of promotional environment. Adv Exp Med Biol, 617: 469-475, 2008.
19. Young LJT: The cleared mammary fat pat and the transplantation of mammary gland morphological structues and cells In: Methods in mammary gland biology and breast cancer research, Ip MM, Asch BB (Eds), pp 67-74, Springer Verlag, New York, 2000.

20. Rajkumar L, Kittrell FS, Guzman RC, Brown PH, Nandi S, Medina D: Hormone-induced protection of mammary tumorigenesis in genetically engineered mouse models. Breast Cancer Res, 9: R12, 2007.

21. Takahashi H, Strutton GM, Parsons PG: Determination of proliferating fractions in malignant melanomas by antiPCNA/cyclin monoclonal antibody. Histopathology, 18: 221-227, 1991.

22. Toillon R, Lagadec C, Page A, Chopin V, Sautière PE, Ricort JM, Lemoine J, Zhang M, Hondermarck H, Le Bourhis X: Proteomics demonstration that normal breast epithelial cells can induce apoptosis of breast cancer cells through insulin-like growth factor-binding protein-3 and maspin. Mol Cell Proteomics, 6: 1239-1247, 2007.

23. Trosko JE: The role of stem cells and gap junctional intercellular communication in carcinogenesis. J Biochem Mol Biol, 36: 43-48, 2003.

24. Campbell LL, Polyak K: Breast tumor heterogeneity: cancer stem cells or clonal evolution? Cell Cycle, 6: 2332-2338, 2007. 